\title{
Dripping to Jetting Transitions in Coflowing Liquid Streams
}

\section{Citation}

Utada, Andrew S., Alberto Fernandez-Nieves, Howard A. Stone, and David A. Weitz. 2007. "Dripping to Jetting Transitions in Coflowing Liquid Streams." Physical Review Letters99 (9): 094502. https://doi.org/10.1103/PhysRevLett.99.094502.

\section{Permanent link}

http://nrs.harvard.edu/urn-3:HUL.InstRepos:41511302

\section{Terms of Use}

This article was downloaded from Harvard University's DASH repository, and is made available under the terms and conditions applicable to Other Posted Material, as set forth at http:// nrs.harvard.edu/urn-3:HUL.InstRepos:dash.current.terms-of-use\#LAA

\section{Share Your Story}

The Harvard community has made this article openly available.

Please share how this access benefits you. Submit a story.

Accessibility 


\title{
Dripping to Jetting Transitions in Coflowing Liquid Streams
}

\author{
Andrew S. Utada, ${ }^{1}$ Alberto Fernandez-Nieves, ${ }^{1,2}$ Howard A. Stone, ${ }^{1}$ and David A. Weitz ${ }^{1,3, *}$ \\ ${ }^{1}$ Harvard School of Engineering and Applied Sciences, Cambridge, Massachusetts 02138, USA \\ ${ }^{2}$ INEST Group, Philip Morris USA, Richmond, Virginia 23224, USA \\ ${ }^{3}$ The Physics Department, Harvard University, Cambridge, Massachusetts 02138, USA
}

(Received 21 October 2006; published 28 August 2007)

\begin{abstract}
A liquid forced through an orifice into an immiscible fluid ultimately breaks into drops due to surface tension. Drop formation can occur right at the orifice in a dripping process. Alternatively, the inner fluid can form a jet, which breaks into drops further downstream. The transition from dripping to jetting is not understood for coflowing fluid streams, unlike the case of drop formation in air. We show that in a coflowing stream this transition can be characterized by a state diagram that depends on the capillary number of the outer fluid and the Weber number of the inner fluid.
\end{abstract}

PACS numbers: 47.61.Jd, 47.20.Dr, 47.27.nf, 47.55.db

A liquid forced through an orifice will ultimately break into drops through one of two mechanisms. At slow flows, the emerging liquid drips from the orifice, whereas at faster flows, the liquid forms a thin stream that breaks into drops away from the orifice; these are the dripping and jetting regimes. This behavior is familiar to anyone who has slowly increased the flow rate of water at a kitchen faucet. In this case, dripping occurs at low flow rates where surface tension causes the water to form drops at the tap; the hanging drops detach when the gravitational force exceeds surface tension forces. At higher flow rates jetting occurs when the inertial forces of the water exceed surface tension forces $[1,2]$. The dripping-to-jetting transition is sharp if the liquid viscosity is large compared to water [2], whereas the transition initially becomes chaotic [1,3-5] before jetting for lower viscosity fluids. The jets eventually break into drops due to the Rayleigh-Plateau instability $[6,7]$.

While both dripping and jetting must occur when a liquid is injected in a second immiscible liquid [8-10], the mechanism of droplet formation changes due to the presence of the surrounding viscous liquid [11-14]. Drop formation has rich dynamics $[15,16]$ that are affected by many parameters such as the average velocities of both liquids, their viscosities and densities, surface tension, and the surface chemistry and device geometry [17]. Twophase drop formation is important to applications in microfluidics $[18,19]$ such as flow focusing $[15,20]$ and in liquidgas systems [21], selective withdrawal for coating particles [22], and extrusion emulsification [23]. Because of its importance, drop formation in two fluids has been widely studied [8-10,24,25]; however, complete control over the two-phase flow behavior requires a detailed understanding of the dripping-to-jetting transition, and a unified view of this transition is still lacking [26].

In this Letter, we use a microcapillary device to study the transition between dripping and jetting in a two-phase coflowing stream. The behavior is characterized by a state diagram that depends on both the capillary number of the outer fluid, $\mathcal{C}_{\text {out }}$, and the Weber number of the inner fluid,
$\mathcal{W}_{\text {in }}$; these parameters describe, respectively, the magnitude of the viscous shear forces from the outer liquid and the inertial forces from the inner liquid compared to surface tension forces. We observe two distinct jetting regimes with significant differences in jet shape and the mechanism controlling drop size.

Our experimental device is made of two coaxially aligned capillary tubes. The inner capillary tube is cylindrical, with a tip tapered to an inner diameter of $d_{\text {tip }} \simeq$ $20 \mu \mathrm{m}$ and an outer diameter of $30 \mu \mathrm{m}$. The outer capillary tube is square; coaxial alignment of the tubes is achieved by matching the outer diameter of the untapered portion of the inner capillary to the inner dimension of the square capillary, $D=1 \mathrm{~mm}$, as shown in Fig. 1(a). At this length scale, which is below the capillary length, the effects of gravity are negligible. Although the flow in the square tube is not axisymmetric, since the tip is centered and $d_{\text {tip }} / D \approx 0.02$, the local flow around the tip should be approximately axisymmetric. For experiments requiring a larger velocity of the outer fluid, we place a second cylindrical capillary with inner diameter of $200 \mu \mathrm{m}$ inside the outer square capillary, surrounding the tip; since this extra capillary has a smaller cross section than the square one, the outer fluid achieves a higher average velocity. Although the surrounding walls are closer in this case, the jet diameter is $\leq d_{\text {tip }}$, and $d_{\text {tip }} / D \approx 0.1$; thus, we expect wall effects to be small. We use deionized water and different viscosity polydimethylsiloxane (PDMS) oils; interchanging the oil and water enables us to vary the viscosity ratio, $\eta_{\text {in }} / \eta_{\text {out }}$, from 0.01 to 10 , where $\eta_{\text {in }}$ and $\eta_{\text {out }}$ are the viscosities of the inner and outer fluids, respectively. The surface tension between PDMS oil and water can be lowered from 40 to $4 \mathrm{mN} / \mathrm{m}$ by adding $60 \mathrm{mM}$ sodium dodecyl sulfate (SDS) to the water. This high concentration of SDS reduces surface tension gradients across the jets.

In coflowing fluids, dripping occurs at low flow rates of both fluids and is characterized by the periodic formation of individual drops that pinch-off from the tip [Fig. 1(b)]. We observe two distinct classes of transitions from drip- 


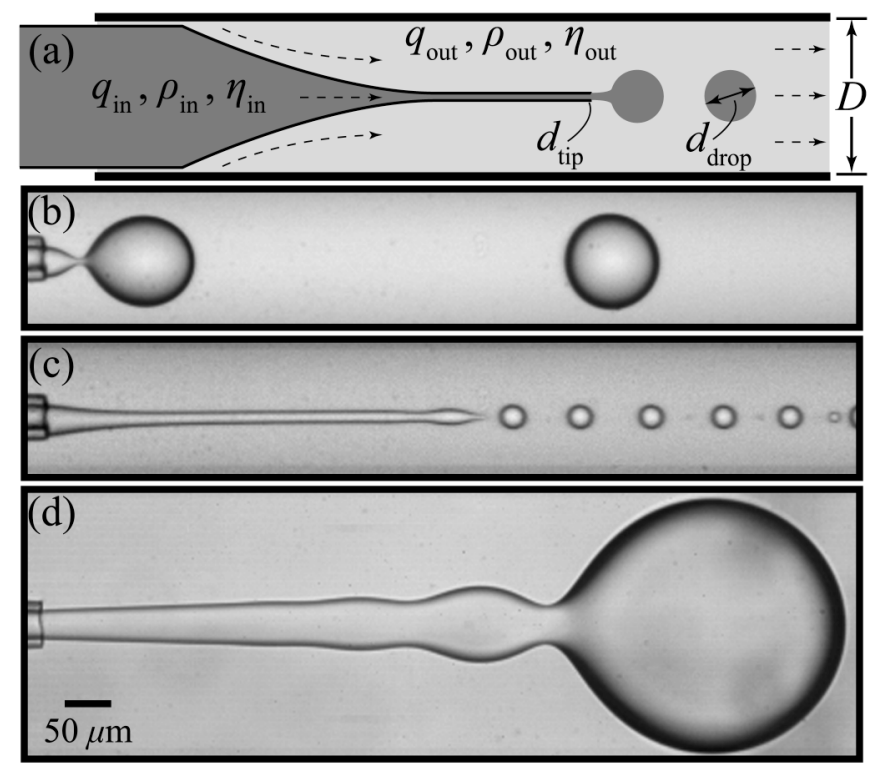

FIG. 1. (a) Device geometry showing the tapered inner capillary in the outer square capillary. (b) Image of the dripping regime. (c) Image of a narrowing jet generated by increasing $q_{\text {out }}$ above a threshold while keeping $q_{\text {in }}$ constant, with $\eta_{\text {in }} / \eta_{\text {out }}=$ 0.1 . (d) Image of a widening jet generated by increasing $q_{\text {in }}$ above a threshold while keeping $q_{\text {out }}$ constant, with $\eta_{\text {in }} / \eta_{\text {out }}=$ 0.1 . Images (b) - (d) were taken with a high-speed camera (walls not shown). Scale bar applies to (b)-(d).

ping to jetting. The first is driven by the flow rate of the outer fluid; as it is increased, drops formed at the tip decrease in size until a jet is formed, whereupon drop breakup occurs downstream at the end of the thin jet [Fig. 1(c)]. The second class of transition is driven by the flow rate of the inner fluid; as it is increased, the dripping drop is pushed downstream and is ultimately pinched off at the end of the resultant jet [Fig. 1(d)].

The first class of dripping-to-jetting transition is characterized by a jet that thins as it moves downstream. In the dripping regime, the diameter of the drop first decreases as the flow rate of the outer liquid, $q_{\text {out }}$, increases. When the drop diameter becomes approximately equal to $d_{\text {tip }}$, there is a spontaneous transition to jetting. The diameter of the jet decreases with distance downstream but ultimately reaches a constant value as shown in Fig. 1(c). Still farther downstream, the jet develops undulations driven by the Rayleigh-Plateau instability; these grow larger and ultimately drive the formation of drops whose diameter is only slightly larger than that of the cylindrical jet itself. In this regime, it is the viscous drag from the flow of the outer fluid that drives drop formation. In the dripping regime, the growing droplet experiences two competing forces: viscous drag pulling it downstream and forces due to surface tension holding it to the tip. Initially, surface tension dominates but the drag forces eventually become comparable as the droplet grows. This force balance is given by: $\eta_{\text {out }} u_{\text {out }} d_{\text {drop }} \sim \gamma d_{\text {tip }}$, where $u_{\text {out }}$ is the mean velocity of the outer liquid and $\gamma$ is the surface tension
[27]. The diameter of the detaching drop decreases as $q_{\text {out }}$ increases; ultimately, a critical shear stress is reached where the emerging liquid is stretched into a narrowing jet [Fig. 1(c)]. The diameter of this jet is initially equal to $d_{\text {tip }}$ and decreases as the liquid moves downstream until the stress gradient across the jet relaxes. This is similar to flow focusing in microfluidic devices $[15,20]$ and in model calculations [28].

We measure the final downstream diameter of the jets and resulting drops from high-speed movies. We compare these data to calculations of the steady-state diameter of the jet, $d_{\text {jet }}$, obtained by solving for the motion of two coaxially flowing liquids with constant shape under Stokes flow: $\eta \nabla^{2} \boldsymbol{u}=\nabla p$, where $p$ is the pressure. By relating the mean velocities of both fluids to the flow rates, we obtain a function [29] where the dominant term is $q_{\text {in }} / q_{\text {out }} \approx$ $2\left(d_{\text {jet }} / D\right)^{2}$. Solving for $d_{\text {jet }} / D$, we obtain the scaled jet diameter as a function of the flow rate ratio. This relation (solid line) agrees well with the measured jet diameters, as shown in Fig. 2.

We can also calculate the drop diameter by assuming that it is related to $d_{\text {jet }}$. For static fluid cylinders suspended in another immiscible liquid, the wavelength of the fastest growing mode of the Rayleigh-Plateau instability is proportional to the diameter of the cylinder itself [30]. In turn, the volume contained in one wavelength of this mode, $\lambda$, on the cylinder, $\pi d_{\text {jet }}^{2} \lambda / 4$, is approximately equal to the volume of the drop that is formed when the cylinder breaks. The most unstable mode is a function of viscosity ratio; in this experiment, $\eta_{\text {in }} / \eta_{\text {out }}=0.1$ and $\lambda=5.48 d_{\text {jet }}$ [30]. We solve for $d_{\text {drop }}$ as a function of $d_{\text {jet }}$, assuming that this argument applies for nearly uniformly translating jets, giving $d_{\text {drop }} \approx 2 d_{\text {jet }}$. The calculated results (dotted line) agree very well with the measured drop sizes, as shown in Fig. 2.

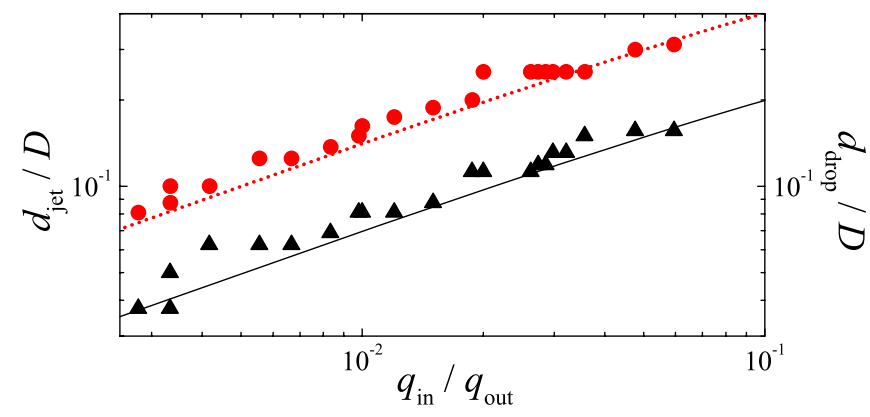

FIG. 2 (color online). Experimentally measured $d_{\text {jet }}(\boldsymbol{\Delta})$ and $d_{\text {drop }}(\bigcirc)$ scaled by $D$ as a function of $q_{\text {in }} / q_{\text {out }}$ for the narrowing jets with $\eta_{\text {in }} / \eta_{\text {out }}=0.1$. The solid line is the prediction from the model for $d_{\text {jet }}$ with no fitting parameters. The dashed line is the predicted result assuming $d_{\text {drop }} \approx 2 d_{\text {jet }}$ from the RayleighPlateau instability as described in the text. The inner and outer Reynolds numbers, $\mathcal{R}_{\text {in }}=\frac{\rho_{\text {in }} d_{\text {tip }} u_{\text {in }}}{\eta_{\text {in }}}$ and $\mathcal{R}_{\text {out }}=\frac{\rho_{\text {out }} D u_{\text {out }}}{\eta_{\text {out }}}$, vary from $\sim 7-70$ and $\sim 15-50$, respectively. 
The second dripping-to-jetting transition is characterized by a dramatically different shape; the jet diameter increases along its length instead of decreasing [Fig. 1(d)]. In this case, the transition from dripping to jetting is no longer sudden; instead, there is an intermediate dripping regime as $q_{\text {in }}$ increases where the growing droplets move downstream while still connected to the fluid in the tip through a fluid neck. However, as soon as the drop pinches off, the neck retracts completely back to the tip, whereupon the process repeats itself. As $q_{\text {in }}$ is increased still further, this intermediate regime transitions fully into jetting, where we define jetting as the incomplete retraction of the fluid neck. The jet ultimately develops undulations that lead to drop formation.

This second class of dripping-to-jetting transition is controlled by different forces and thus exhibits different behavior. The control parameter $q_{\text {in }}$ does not affect drop size in the dripping regime; instead drop size depends on the viscous drag, which depends on $q_{\text {out }}$. Since $q_{\text {out }}$ is held constant, $q_{\text {in }}$ affects how quickly the droplets reach the critical size where the viscous drag and surface tension forces balance each other. However, $q_{\text {in }}$ is proportional to the momentum of the inner fluid, which acts cooperatively with the viscous drag from the outer fluid to drive the transition to the intermediate regime, where the droplet moves downstream, though still connected to the fluid in the tip through a fluid neck. After the drop pinches off, surface tension pulls the neck completely back into the tip. At even larger $q_{\text {in }}$, the inertia of the injected inner liquid becomes sufficiently large that the position of drop growth and pinch off is pushed away from the tip; the large inertial forces within the jet prevent the fluid neck from retracting completely back to the tip after drop pinch off. There is, however, a slight retraction at the end of the jet due to surface tension, which causes the end to become spherical and move slightly upstream. Viscous drag from the outer fluid then pulls the growing droplet downstream beginning the process again. The significant shape differences between these jets and the thinning jets [see Figs. 1(c) and 1(d)] are due to the large velocity difference between the inner and outer liquids. For widening jets, the inner liquid is injected at a much larger velocity than the average velocity of the outer liquid. The large shear at the interface due to the velocity difference decelerates the jet, causing it to widen.

The large qualitative shape differences between the widening and the thinning jets indicate a different balance of dominant forces. These differences even change the mechanism controlling drop size, which is highlighted by the fact that in the widening regime $d_{\text {drop }} \neq 2 d_{\text {jet }}$. Representative data sets illustrate trends in drop size as a function of $q_{\text {in }}$ and $u_{\text {out }}$, as shown in the upper inset of Fig. 3. To elucidate the drop-size scaling from these scattered data, we use mass conservation to relate drop diameter to the control parameters: the volume of a drop is $V=q_{\text {in }} \tau$, where $\tau$ is the drop formation time. Drop for-

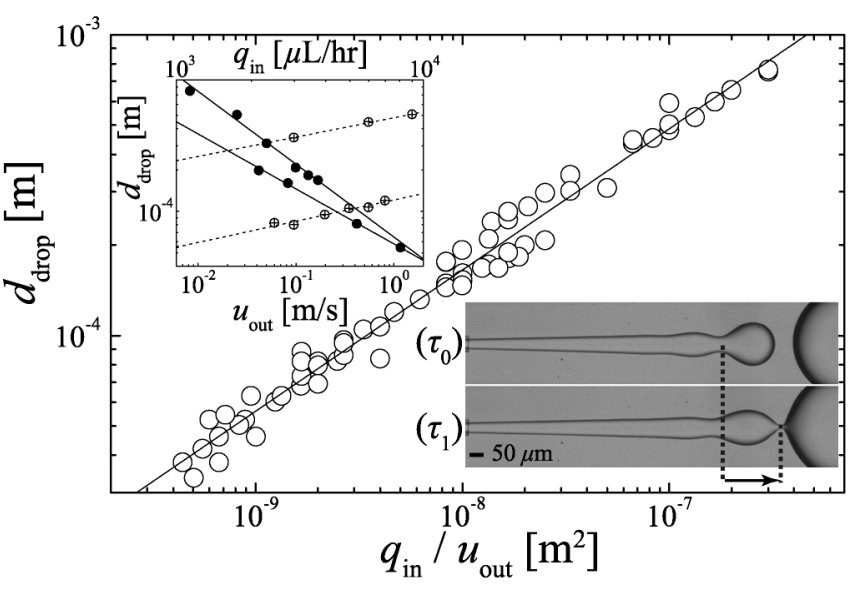

FIG. 3. Measured drop diameter $(\bigcirc)$ as a function of $q_{\text {in }} / u_{\text {out }}$ for widening jets with $\eta_{\text {in }} / \eta_{\text {out }}=0.1$. The slope of the best fit, shown as the solid line, is 0.46. Upper inset: Drop diameter as a function of $u_{\text {out }}$, for constant $q_{\text {in }}(\oplus)$ and as a function of $q_{\text {in }}$, for constant $q_{\text {out }}(\mathbf{O})$. Lower inset: Image (top) just after drop pinchoff, where the jet has retracted due to surface tension and just before the next drop pinches-off (bottom). The end of the jet travels a distance indicated by the arrow in this time interval. Here, $\mathcal{R}_{\text {in }}$ and $\mathcal{R}_{\text {out }}$ vary from $\sim 60-200$ and $\sim 1-60$, respectively.

mation encompasses three steps: advection and growth of the droplet at the end of the jet, drop pinchoff, and finally retraction of the neck. The advection of the growing droplet by the viscous drag is the slowest of these steps; images illustrating the distance the jet is advected are shown in the lower inset of Fig. 3. Since the velocity of the inner liquid is much larger than the advection velocity, we expect $\tau$ to scale as the advected distance divided by the advection velocity. This distance is typically $\sim d_{\text {drop }}$ while the velocity is $\sim u_{\text {out }}$, which leads to $d_{\text {drop }} \sim\left(q_{\text {in }} / u_{\text {out }}\right)^{1 / 2}$. This agrees with the exponent of 0.46 determined from a fit of the data for $\eta_{\text {in }} / \eta_{\text {out }}=0.1$, as shown by the solid line in Fig. 3. While we limit our discussion of drop scaling to $\eta_{\text {in }} / \eta_{\text {out }}=0.1$, this result is notable because drop size is set by viscous drag forces on the growing droplet, whereas in the limit of $\eta_{\text {in }} / \eta_{\text {out }} \gg 1$, jet and drop diameters are set solely by the tip diameter, regardless of the jet velocity.

To unify these two distinct jetting transitions, we consider the balance of forces on the droplet immediately before the dripping-to-jetting transition. In the first regime, where the jets become thinner, the viscous shear stresses on the drop must be balanced by surface tension; this balance is given by the capillary number of the outer fluid, $\mathcal{C}_{\text {out }}=$ $\eta_{\text {out }} u_{\text {out }} / \gamma$, at the transition. When $\mathcal{C}_{\text {out }}$ is small, surface tension dominates, forcing the system to drip. By contrast, when $\mathcal{C}_{\text {out }} \geq O(1)$, the shear stress on the droplet is large enough to overcome surface tension, leading to jetting. In the second regime, where the jets become wider, the inertial forces of the inner fluid must be balanced by the surface tension forces; this balance is given by the inner Weber number, $\mathcal{W}_{\text {in }}=\rho_{\text {in }} d_{\text {tip }} u_{\text {in }}^{2} / \gamma$. When $\mathcal{W}_{\text {in }}$ is small, surface tension dominates, forcing the system to drip. By contrast, 


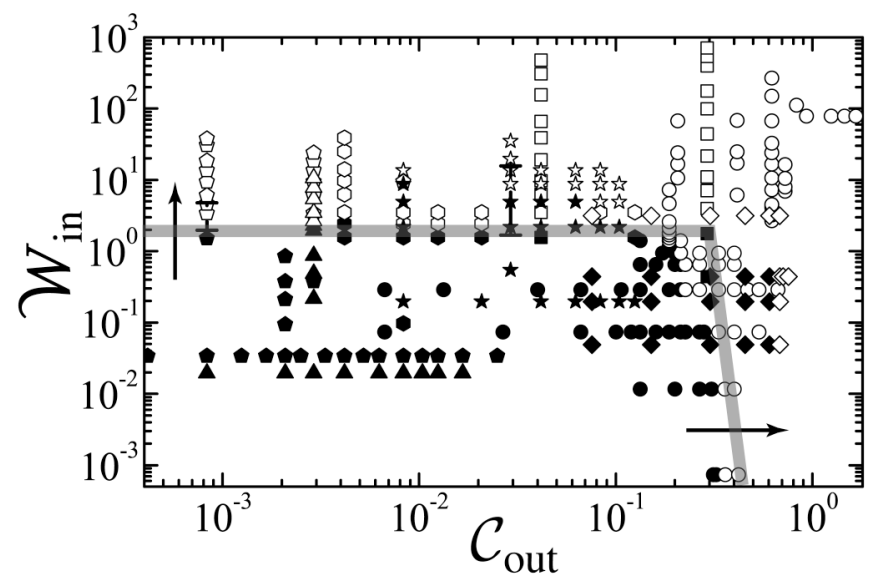

FIG. 4. State diagram of the dripping-to-jetting transition in a coflowing stream as a function of $\mathcal{C}_{\text {out }}$ and $\mathcal{W}_{\text {in }}$. Filled symbols represent dripping while open symbols represent jetting. Each shape is a different viscosity ratio, surface tension, or geometry. Surface tension is $40 \mathrm{mN} / \mathrm{m}$ unless otherwise stated. Square: $\eta_{\text {in }} / \eta_{\text {out }}=0.01$. Diamond: $\eta_{\text {in }} / \eta_{\text {out }}=0.01$, with the extra capillary tube to increase $u_{\text {out }}$. Hexagon: $\eta_{\text {in }} / \eta_{\text {out }}=0.01$. Circle: $\eta_{\text {in }} / \eta_{\text {out }}=0.01$, with the extra capillary tube to increase $u_{\text {out }}$. Pentagon: $\eta_{\text {in }} / \eta_{\text {out }}=1$. Triangle: $\quad \eta_{\text {in }} / \eta_{\text {out }}=10$. Star: $\eta_{\text {in }} / \eta_{\text {out }}=10$ and $\gamma=4 \mathrm{mN} / \mathrm{m}$. Typical error bars for different surface tensions are shown.

when $\mathcal{W}_{\text {in }} \geq O(1)$, the inertial forces are large enough to overcome surface tension, leading to jetting. To generalize this result, we vary the viscosity ratio between 0.01 and 10 and lower surface tension from 40 to $4 \mathrm{mN} / \mathrm{m}$ for $\eta_{\text {in }} / \eta_{\text {out }}=10$. We plot a state diagram showing dripping (closed symbols) and jetting (open symbols) as a function of $\mathcal{C}_{\text {out }}$ and $\mathcal{W}_{\text {in }}$. Dripping occurs when both $\mathcal{C}_{\text {out }}$ and $\mathcal{W}_{\text {in }}$ are small; however, there is a distinct transition when either increases, as shown by the wide gray line in Fig. 4. The good collapse of the data reveals that in the coflowing stream the sum of the forces exerted on the droplet must ultimately overcome surface tension forces to cause the transition; this can be summarized by $\mathcal{C}_{\text {out }}+\mathcal{W}_{\text {in }} \approx O(1)$.

Our results identify the different jetting responses and highlight the importance of the coflowing viscous liquid in the dripping-to-jetting transition. For our experiments, the two jetting regimes result from a different balance of dominant forces. However, the two regimes can be integrated into a single picture that ultimately depends on the ability of these forces to deform the emerging liquid from a spherical drop to a cylindrical jet. In general, we expect the flow response to depend on 5 dimensionless parameters, which can be chosen as $\left(\mathcal{W}_{\text {in }}, \mathcal{C}_{\text {out }}, \mathcal{R}_{\text {in }}, \mathcal{R}_{\text {out }}, u_{\text {in }} / u_{\text {out }}\right)$, where $\mathcal{R}$ is the Reynolds number. We find that all of our data are captured by $\left(\mathcal{W}_{\text {in }}, \mathcal{C}_{\text {out }}\right)$ for moderate $\mathcal{R}$. Thus our work provides a general framework for understanding a wide range of behavior, though clearly there are regions of the parameter space yet unexplored. A better understanding of this transition will provide a higher degree of control over emulsions generated with shear flows. Moreover, although we consider only Newtonian fluids, the basis for this transition may also clarify similar processes involving non-Newtonian fluids.

This work was supported by the NSF (No. DMR0602684) and the Harvard MRSEC (No. DMR-0213805). A. F.-N. thanks the University of Almeria.

*weitz@seas.harvard.edu

[1] C. Clanet and J.C. Lasheras, J. Fluid Mech. 383, 307 (1999).

[2] B. Ambravaneswaran et al., Phys. Rev. Lett. 93, 034501 (2004).

[3] R. Shaw, The Dripping Faucet as a Model Chaotic System (Aerial, Santa Cruz, CA, 1984).

[4] B. Ambravaneswaran, S. D. Phillips, and O. A. Basaran, Phys. Rev. Lett. 85, 5332 (2000).

[5] P. Coullet, L. Mahadevan, and C. S. Riera, J. Fluid Mech. 526, 1 (2005).

[6] J. Plateau, Acad. Sci. Bruxelles Mém. 23, 5 (1849).

[7] L. Rayleigh, Proc. R. Soc. London 29, 71 (1879).

[8] G. F. Scheele and B. J. Meister, AIChE J. 14, 9 (1968).

[9] B. J. Meister and G. F. Scheele, AIChE J. 15, 689 (1969); 15, 700 (1969).

[10] J.R. Richards, A. N. Beris, and A.M. Lenhoff, Phys. Fluids 7, 2617 (1995).

[11] D. F. Zhang and H. A. Stone, Phys. Fluids 9, 2234 (1997).

[12] J. R. Lister and H. A. Stone, Phys. Fluids 10, 2758 (1998).

[13] W. W. Zhang and J. R. Lister, Phys. Rev. Lett. 83, 1151 (1999).

[14] I. Cohen et al., Phys. Rev. Lett. 83, 1147 (1999).

[15] S. L. Anna, N. Bontoux, and H. A. Stone, Appl. Phys. Lett. 82, 364 (2003).

[16] P. Garstecki, M. J. Fuerstman, and G. M. Whitesides, Phys. Rev. Lett. 94, 234502 (2005).

[17] J. Eggers, Rev. Mod. Phys. 69, 865 (1997).

[18] M. Joanicot and A. Ajdari, Science 309, 887 (2005).

[19] H. A. Stone, A. D. Stroock, and A. Ajdari, Annu. Rev. Fluid Mech. 36, 381 (2004).

[20] A. M. Gañán-Calvo and J. M. Gordillo, Phys. Rev. Lett. 87, 274501 (2001).

[21] B. K.H. Yen et al., Angew. Chem., Int. Ed. 44, 5447 (2005).

[22] I. Cohen et al., Science 292, 265 (2001).

[23] G. T. Vladisavljevic and R. A. Williams, Adv. Colloid Interface Sci. 113, 1 (2005).

[24] B. J. Meister and G. F. Scheele, AIChE J. 13, 682 (1967).

[25] J.R. Richards, A.N. Beris, and A.M. Lenhoff, Phys. Fluids A 5, 1703 (1993); J. R. Richards, A. M. Lenhoff, and A. N. Beris, Phys. Fluids 6, 2640 (1994).

[26] C. Cramer, P. Fischer, and E. J. Windhab, Chem. Eng. Sci. 59, 3045 (2004).

[27] P. B. Umbanhowar, V. Prasad, and D. A. Weitz, Langmuir 16, 347 (2000).

[28] J. Meyer and D. Weihs, J. Fluid Mech. 179, 531 (1987).

[29] A.S. Utada et al., Science 308, 537 (2005).

[30] S. Tomotika, Proc. R. Soc. A 150, 322 (1935). 